\title{
RANDOM MUTAGENESIS OF HUMAN SERINE RACEMASE REVEALS RESIDUES IMPORTANT FOR THE ENZYMATIC ACTIVITY
}

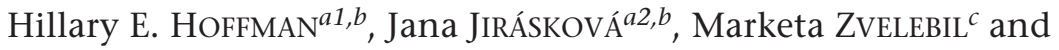 \\ Jan KONVALINKA ${ }^{a 3, b, *}$ \\ ${ }^{a}$ Gilead Sciences and IOCB Research Center, Institute of Organic Chemistry and Biochemistry, \\ Academy of Sciences of the Czech Republic, v.v.i., Flemingovo nám. 2, 16610 Prague 6, \\ Czech Republic; e-mail: ${ }^{1}$ hehoffman@gmail.com, ${ }^{2}$ jana.jiraskova@uochb.cas.cz, \\ ${ }^{3}$ konval@uochb.cas.cz \\ ${ }^{b}$ Department of Biochemistry, Faculty of Science, Charles University in Prague, \\ Hlavova 8, 12843 Prague 2, Czech Republic \\ ${ }^{c}$ Breakthrough Breast Cancer Research Centre, The Institute of Cancer Research, \\ 237 Fulham Road, London SW3 6JB, United Kingdom
}

Received January 13, 2010 Accepted January 13, 2010 Published online February 8, 2010

Human serine racemase (hSR) is a cytosolic pyridoxal-5'-phosphate dependent enzyme responsible for production of D-serine in the central nervous system. D-Serine acts as an endogenous coagonist of $\mathrm{N}$-methyl-D-aspartate receptor ion channels. Increased levels of D-serine have been linked to amyotrophic lateral sclerosis and Alzheimer's disease, indicating that SR inhibitors might be useful tools for investigation or treatment of neurodegenerative diseases. However, despite hSR's promise as a therapeutic target, relatively few specific inhibitors have been identified, which is due in part to the lack of a three-dimensional structure of the enzyme. Here, we present a strategy for the generation and screening of random hSR mutants. From a library of randomly mutated hSR variants, twenty-seven soluble mutants were selected, expressed, and evaluated for enzymatic activity. Taking three carefully characterized mutants as an example, we show how this strategy can be used to pinpoint structurally and functionally important residues. In particular, we identify S84 and P111 as residues crucial for hSR activity and C217 and $\mathrm{K} 221$ as residues important for binding of the $\mathrm{Mg}^{2+}$ cofactor as well as for overall stability of the enzyme.

Keywords: D-Serine; Serine racemase; Error-prone PCR; Random mutagenesis; ThermoFluor assay; Enzymes; Racemases; Enzyme engineering; In vitro evolution.

Eukaryotic serine racemase (SR; EC 5.1.1.18) is a cytosolic pyridoxal-5'-phosphate (PLP) dependent enzyme responsible for the production of D-serine from L-serine and vice versa. In the mammalian central nervous system (CNS), $\mathrm{D}$-serine acts as an endogenous coagonist of $\mathrm{N}$-methyl-D-aspartate (NMDA) receptors, which are involved in glutamate-mediated excitatory neurotrans- 
mission. In addition to its racemase activity, SR is also involved in serine metabolism by acting as a $\beta$-eliminase, converting L-serine $\mathrm{C}^{1-3}$, and to a lesser extent D-serine ${ }^{4}$, to pyruvate, with concomitant ammonia release. Both the racemization and elimination activities of mammalian SR are upregulated by divalent cations $\mathrm{s}^{1,5}, \mathrm{ATP}^{3}$, and reducing agents ${ }^{6}$.

Aberrant regulation of NMDA receptor mediated pathways has been implicated in a variety of neuropathologies, and excess D-serine production and overexpression of SR have been linked to Alzheimer's disease ${ }^{7}$ and amyotrophic lateral sclerosis ${ }^{8}$. While high-affinity NMDA receptor blockers are in clinical use, treatment of patients with these drugs is often accompanied by undesirable side effects such as hallucinations. In recent years, there has been a movement towards development of low-affinity NMDA receptor blockers that are both therapeutically effective and better tolerated by patients ${ }^{9,10}$. In a similar manner, SR inhibitors might offer a more "gentle" approach to decrease NMDA receptor overactivation. Recently, Inoue and coworkers showed that amyloid- $\beta$-peptide mediated excitotoxicity is abrogated in SR knock-out mice ${ }^{11}$, providing compelling evidence for the potential utility of SR inhibitors as therapeutic agents. However, very few competitive SR inhibitors have been described to date, and the most potent of these have $K_{i}$ values in the micromolar range ${ }^{12,13}$.

In order to design specific active-site-directed ligands with high potency and in order to gain a greater understanding of the catalytic and control mechanisms of this enzyme, it would be useful to determine the 3D structure of mammalian SR. While the structure of a SR orthologue from the fission yeast Schizosaccharomyces pombe $\left(35.1 \%{ }^{14}\right.$ or $40 \%{ }^{15}$ sequence identity to human SR) has been solved ${ }^{14,16}$, the structure of a mammalian SR orthologue remains elusive. We therefore set out to establish a strategy for the generation and screening of human SR mutants in hopes of gaining insight into the structure-function relationships within the enzyme.

We have recently reported the heterologous expression of human SR (hSR) in Escherichia coli and purification and characterization of the recombinant enzyme ${ }^{17}$. In this work, we subjected the hSR gene to random mutagenesis by error-prone PCR and generated a plasmid library. The library was transformed into E. coli, and colonies expressing soluble hSR were detected with a dot-blot assay. For the initial analysis, twenty-seven soluble mutants were selected, expressed on a small-scale, partially purified, and evaluated for L-serine racemization and $\beta$-elimination activity. Three of these mutants - C217S, K221E, and S84G/P111L - were purified on a larger scale and analyzed in terms of thermal stability and activity. Multiple sequence alignment of 8 eukaryotic serine racemases and previously described homology 
model $s^{17,18}$ were used to complement the experimental data and aid in assessing the potential function of the mutated residues.

\section{EXPERIMENTAL}

\section{Abbreviations Used}

CNS, central nervous system; DTT, 1,4-dithiothreitol; EP PCR, error-prone polymerase chain reaction; HEPES, 4-(2-hydroxyethyl)-1-piperazineethanesulfonic acid; IMAC, immobilized metal affinity chromatography; NiNTA, nickel nitrilotriacetic acid; NMDA, N-methylD-aspartate; PLP, pyridoxal-5'-phosphate; SR, serine racemase (hSR, human SR; mSR, mouse $\mathrm{SR}) ; \mathrm{TAE}$, tris-acetate ethylenediaminetetraacetic acid.

\section{Error-Prone Polymerase Chain Reaction}

The hSR gene was amplified under error-prone conditions from the plasmid pMPMhSR6His ${ }^{17}$. PCR reactions were set up according to two established protocols ${ }^{19,20}$. In the first protocol, each reaction contained the template, $1 \mu \mathrm{M}$ forward primer, $1 \mu \mathrm{M}$ reverse primer, $4.2 \mathrm{~mm}$ $\mathrm{MgCl}_{2}, 0.5 \mathrm{~mm} \mathrm{MnCl}_{2}$ (molecular biology grade, Sigma), $1 \mu \mathrm{l}$ Taq DNA polymerase (New England Biolabs), and $1 X$ Standard Taq (Mg-free) buffer. Four reactions were set up in parallel: in each, one dNTP was present at $3.4 \mathrm{~mm}$, while the other three were added to final concentrations of $0.2 \mathrm{~mm}$ each. In the second protocol, the reactions contained the template, $1 \mu \mathrm{m}$ each primer, $5 \mathrm{~mm} \mathrm{MgCl}_{2}, 1 \mu \mathrm{l}$ Taq DNA polymerase, 1X Standard Taq (Mg-free) buffer, $0.2 \mathrm{~mm}$ dITP (New England Biolabs), $20 \mu \mathrm{m}$ of one dNTP, and $0.2 \mathrm{~mm}$ of each of the three other dNTPs. The same thermal cycles were used for both protocols: an initial denaturation period of $5 \mathrm{~min}$ at $94{ }^{\circ} \mathrm{C}$, followed by 16 cycles of $1 \mathrm{~min}$ at $94{ }^{\circ} \mathrm{C}, 1 \mathrm{~min}$ at $55{ }^{\circ} \mathrm{C}$, and $3 \mathrm{~min}$ at $72{ }^{\circ} \mathrm{C}$. The PCR products were run on a $1 \% \mathrm{TAE} /$ agarose gel, and the appropriatelysized bands (approximately $1 \mathrm{kbp}$ ) were excised and purified with the QiaQuik Gel Extraction Kit (Qiagen) according to the manufacturer's instructions. The purified PCR products were combined and digested with $\mathrm{Ndel}$ and $\mathrm{X}$ hol, then run on a $1 \% \mathrm{TAE} /$ agarose gel, excised, and again purified with the QiaQuik kit. The mixture was ligated into pMPM-A4-2 ${ }^{12}$ digested with $\mathrm{Ndel} / \mathrm{Xhol}$ using T4 DNA ligase, and $5 \mu$ of the ligation reactions were transformed into $\mathrm{CaCl}_{2}$-competent E. coli $\mathrm{MC} 1061$.

\section{Solubility Screening}

The mutants were checked for soluble SR expression using a dot-blot protocol based on the work of Cornvik et al. ${ }^{21}$. Colonies were lifted using a nitrocellulose membrane, and the membrane was placed, colonies facing up, onto an LB/agar/ampicillin plate spread with $10 \mathrm{~mm}$ L-arabinose. SR expression was induced at $37{ }^{\circ} \mathrm{C}$ for $4 \mathrm{~h}$. The nitrocellulose membrane with the colonies was then placed on top of a second nitrocellulose membrane and a piece of filter paper soaked in lysis buffer (50 mm phosphate buffer, $\mathrm{pH} 8.0,300 \mathrm{~mm} \mathrm{NaCl}, 20 \mu \mathrm{M}$ PLP, $1 \mathrm{~mm} \mathrm{M} \mathrm{gCl}$, $5 \mathrm{~mm}$ 2-mercaptoethanol, $10 \mathrm{~mm}$ imidazole, $1 \mathrm{mg} / \mathrm{ml}$ lysozyme). The cells were subjected to 3 freeze-thaw cycles ( $10 \mathrm{~min}$ at $-70{ }^{\circ} \mathrm{C}$ followed by $10 \mathrm{~min}$ at $37^{\circ} \mathrm{C}$ ). The upper nitrocellulose membrane was then discarded, while the lower membrane was incubated with Casein Blocker (Pierce, Rockford, Illinois) for $1 \mathrm{~h}$, followed by overnight incubation with anti-SR antibody (1:500 dilution, BD Biosciences and/or antibodies produced in-house (un- 
published results)). The membrane was then washed with PBS containing 0.05\% TWEEN 20, incubated with horseradish peroxidase conjugated goat antimouse immunoglobulin (1:10,000, Pierce), then washed again. The blot was developed with SuperSignal WestPico chemiluminescence substrate (Pierce).

\section{Mini-Purification and Characterization}

Mutants expressing soluble SR variants were grown overnight in $10 \mathrm{ml}$ cultures of autoinducing ZYP-5052 broth $^{22}$ supplemented with $0.05 \%$ arabinose and $100 \mu \mathrm{M}$ ampicillin. The cultures were spun down, and the cell pellets were resuspended in lysis buffer and transferred to microcentrifuge tubes. After $30 \mathrm{~min}$ incubation at room temperature, the cells were sonicated $3 \times 20 \mathrm{~s}$. The insoluble fraction was pelleted by centrifugation at $16,000 \mathrm{~g}, 4{ }^{\circ} \mathrm{C}$, $20 \mathrm{~min}$. The supernatant was then added to $100 \mu \mathrm{l}$ NiNTA beads pre-equilibrated in wash 1 buffer (same as lysis buffer but without lysozyme). After $30 \mathrm{~min}$ gentle rocking at room temperature, the NiNTA beads were allowed to settle to the bottom of the tube, and the "flow-through" fraction was removed. The beads were then washed once with $1 \mathrm{ml}$ of wash 1 buffer and twice with $1 \mathrm{ml}$ of wash2 buffer (same as wash 1 but with $30 \mathrm{~mm}$ imidazole). SR bound to beads was eluted with $500 \mu$ l elution buffer (containing $250 \mathrm{~mm}$ imidazole).

The eluted fractions were run on 12\% SDS-PAGE and stained with Coomassie Brilliant Blue, and the purity of the preparations and quantity of SR present were accessed with ImageJ software (http://rsb.info.nih.gov/ij). Purified samples were then dialyzed against $100 \mathrm{~mm}$ HEPES, pH 8.0, containing $1 \mathrm{~mm} \mathrm{MgCl}_{2}$ and $10 \mu \mathrm{M}$ PLP.

For activity reactions, ATP was added to the dialyzed samples to a final concentration of $1 \mathrm{~mm}$, and DTT was added to $5 \mathrm{~mm}$. Reactions were started by addition of $5 \mathrm{~mm}$ L-serine. After incubation at $37^{\circ} \mathrm{C}$ for approximately $2 \mathrm{~h}$, the reactions were quenched with perchloric acid and processed as described below. DNA for sequencing was prepared by growing single colonies overnight in $5 \mathrm{ml}$ LB/ampicillin. Plasmids were purified from the cultured bacteria using the Spin Miniprep Kit from Qiagen. Sequencing was performed at the sequencing lab of the Faculty of Science of Charles University (Czech Republic) or at Macrogen (South Korea).

\section{Large-Scale Purification}

Plasmids were transformed into E. coli MC1061 and plated on LB/agar/ampicillin plates. Colonies were washed into Terrific Broth (12 g/l NZ amine, $24 \mathrm{~g} / \mathrm{l}$ yeast extract, $0.4 \%$ glycerol, $0.017 \mathrm{M} \mathrm{KH}_{2} \mathrm{PO}_{4}, 0.072 \mathrm{M} \mathrm{K}_{2} \mathrm{HPO}_{4}$ ) containing $100 \mu \mathrm{g} / \mathrm{l}$ ampicillin, and cultures were grown at $250-270 \mathrm{rpm}$ and $37{ }^{\circ} \mathrm{C}$ until the $\mathrm{OD}_{600}$ reached $0.4-0.6$. SR expression was then induced by addition of L-arabinose to a final concentration of $1 \mathrm{~mm}$. After additional $4 \mathrm{~h}$ at $37^{\circ} \mathrm{C}$, cultures were harvested by centrifugation.

Lysis buffer was supplemented with Complete Mini EDTA-free Protease Inhibitor Tablets (Roche), and $5 \mathrm{ml}$ were added per gram cells wet weight. After $20 \mathrm{~min}$ at ambient temperature, an additional $1 \mathrm{mg}$ lysozyme per gram cells and DNAsel (0.1 mg per $100 \mathrm{ml}$ lysate) were added, and the mixture was incubated at room temperature for another $20 \mathrm{~min}$. The samples were then sonicated $3 \times 20 \mathrm{~s}$. The insoluble fraction was pelleted by centrifugation at $18,000 \mathrm{~g}, 4{ }^{\circ} \mathrm{C}, 20 \mathrm{~min}$. The purification was carried out on an ÄKTAprime FPLC (GE Healthcare). The soluble fraction was loaded onto NiNTA FastFlow resin (Qiagen) equilibrated with wash1 buffer. The column was washed with wash 1 buffer until the $A_{280}$ stabilized, then washed with wash2 buffer for a minimum of 10 column volumes or until the 
$\mathrm{A}_{280}$ stabilized. Elution was carried out with a gradient of 30-250 mm imidazole over $30 \mathrm{ml}$; 2-ml fractions were collected. The fractions were analyzed by SDS-PAGE, and SR-rich fractions were pooled and extensively dialyzed against QA buffer (20 mM triethanolamine, $\mathrm{pH}$ 7.4, $20 \mu \mathrm{M}$ PLP, $1 \mathrm{~mm} \mathrm{MgCl}_{2}, 0.1 \mathrm{~mm} \mathrm{DTT}, 0.02 \% \mathrm{NaN}_{3}$ ).

\section{Activity Assays}

SR activity was analyzed in a pH 8.0 reaction buffer consisting of $100 \mathrm{~mm}$ HEPES-NaOH, $10 \mu \mathrm{m}$ PLP, $1 \mathrm{~mm} \mathrm{MgCl}_{2}$, and $1 \mathrm{~mm}$ ATP. Except where otherwise indicated, DTT was included at a concentration of $5 \mathrm{~mm}$. For initial screening, a substrate concentration of $5 \mathrm{~mm}$ L-serine was used and reactions were started by addition of substrate. For determination of specific activity, $4 \mathrm{~mm}$ L-serine (Figs $5 \mathrm{a}$ and $5 \mathrm{c}$ ) or $10 \mathrm{~mm}$ D-serine (Figs $5 \mathrm{~b}$ and $5 \mathrm{~d}$ ) was used and reactions were started by addition of enzyme. Protein concentrations were determined by Bio-Rad Protein Assay (Bio-Rad Laboratories, California, USA). The enzymatic reactions were quenched with perchloric acid to precipitate SR, then neutralized with potassium hydroxide. For racemization analysis, the reaction mixture was derivatized by 1-fluoro-2,4-dinitrophenyl-5-L-alanine amide (Marfey's reagent, Pierce, USA) and resolved using reversed-phase HPLC as described ${ }^{1}$. Pyruvate formation was determined by derivatization of reaction mixtures with 2,4-dinitrophenylhydrazine followed by resolution on reversed-phase HPLC as described $^{12}$.

\section{Gel Filtration}

Gel filtration was carried out on a Superdex200 HR 10/30 column (Pharmacia, Uppsala, Sweden) in QA buffer supplemented with $1 \mathrm{~mm}$ DTT and $150 \mathrm{~mm} \mathrm{NaCl}$. The following proteins were used as calibration standards: ferritin (440 kDa), aldolase (158 kDa), bovine serum albumin (67 kDa), ovalbumin (43 kDa), and chymotrypsinogen (25 kDa).

\section{ThermoFluor Thermal Stability Assay}

Reactions consisted of $35 \mu \mathrm{l}$ buffer solution, $10 \mu \mathrm{l}$ hSR $(1.6-2 \mathrm{mg} / \mathrm{ml})$, and $5 \mu \mathrm{l} 4 \mathrm{X}$ Sypro Orange dye (Sigma Aldrich). The total reaction volume was $50 \mu \mathrm{l}$, and the final buffer composition was $100 \mathrm{~mm}$ HEPES ( $\mathrm{pH}$ 8.0) and $10 \mu \mathrm{M}$ PLP. Where indicated, the buffer was supplemented with $1 \mathrm{~mm}$ ATP, $1 \mathrm{~mm} \mathrm{MgCl}_{2}, 5 \mathrm{~mm}$ DTT, or a combination thereof. The reactions were heated from room temperature to $90{ }^{\circ} \mathrm{C}$ in a Light Cycler 480 Real-Time PCR machine (Roche). The excitation filter was set to $465 \mathrm{~nm}$ and the emission filter to $580 \mathrm{~nm}$, and fluorescence measurements were recorded continuously. The data was analyzed with the accompanying software, and melting temperatures were calculated manually from the first derivatives.

\section{Sequence Alignment}

We performed multiple sequence alignment of SRs from 8 eukaryotic organisms (Homo sapiens, Bos taurus, Rattus norvegicus, Mus musculus, Arabidopsis thaliana, Hordeum vulgare, Schizosaccharomyces pombe, and Saccharomyces cerevisiae) using T-Coffee ${ }^{23}$. 


\section{RESULTS AND DISCUSSION}

Generation of a Library of Random Human Serine Racemase Mutants

Random mutagenesis is a valuable tool for protein modification, and a plethora of methods for producing mutated genes exists, including mutator E. coli strains, chemical reagents such as hydroxylamine, and error-prone polymerase chain reaction. A recent critical comparison of these methods suggests that EP PCR exhibits the widest operational range and highest degree of controllability ${ }^{24}$. Furthermore, the authors suggest the use of a combination of EP PCR protocols in order to ensure a high level of diversity ${ }^{24}$.

In this work, the plasmid pMPMhSR6His, which encodes hSR with a C-terminal 6-histidine tag ${ }^{17}$, was used as a template for EP PCR as described in detail in Experimental. We aimed for a mutation rate resulting in an average of 1-4 amino acid changes per mutant, and we employed two EP PCR protocols. The first, which involved varying the concentrations of the individual dNTPs and the addition of $\mathrm{MnCl}_{2}$ to the reaction mixture ${ }^{19}$, resulted in a mutation rate of 2.3 mutations per 1000 base pairs (the hSR gene consists of 1040 base pairs), with $83 \%$ transition mutations (purine to purine or pyrimidine to pyrimidine) and $13 \%$ transversions (purine to pyrimidine or vice versa). Single nucleotide insertions or del etions were less frequent, comprising $4 \%$ of the total (all percentages based on sequencing data from 10,033 base pairs). The second protocol involved the use of various concentrations of dNTPs and the addition of dITP to the reaction mixture ${ }^{20}$. This protocol resulted in a slightly higher mutation rate of 8.5 mutations per 1000 base pairs. Transition mutations were again favored, accounting for $80.7 \%$ of the total, while transversion mutations accounted for $19.3 \%$. No insertions or deletions were observed in the 13,468 base pairs sequenced. The randomly mutated PCR products generated by both protocols were pooled, then digested with $\mathrm{Ndel}$ and $\mathrm{Xhol}$ and ligated into the arabinose-inducible vector PMPM-A4-2 ${ }^{12}$ to produce a plasmid library.

\section{Library Screening}

The plasmid library was transformed into E. coli MC1061. A colony dot-blot assay was employed to select colonies expressing soluble hSR variants, as illustrated in Fig. 1a. The assay was based on the colony filtration blot strategy reported by Cornvik et al. ${ }^{21}$, and procedures are described in detail in Experimental. Briefly, transformants were transferred onto a nitrocellulose membrane, and SR expression was induced with $10 \mathrm{~mm}$ L-arabinose. Fol- 
lowing induction, the membrane with colonies bound was placed on top of a second nitrocellulose membrane and a piece of filter paper soaked in lysis buffer. The cells were lysed, which resulted in migration of the soluble proteins to the lower nitrocellulose membrane, while the insoluble fraction remained on the upper membrane. The lower membrane was then probed with anti-SR antibody in order to identify colonies producing soluble SR variants. This method appears to be quite robust, and we did not identify any false positives. However, in cases in which mutations occur within the antibody recognition site, the possibility of obtaining false negatives exists. We controlled for this possibility by probing the membranes with a mixture of anti-SR antibodies.

a
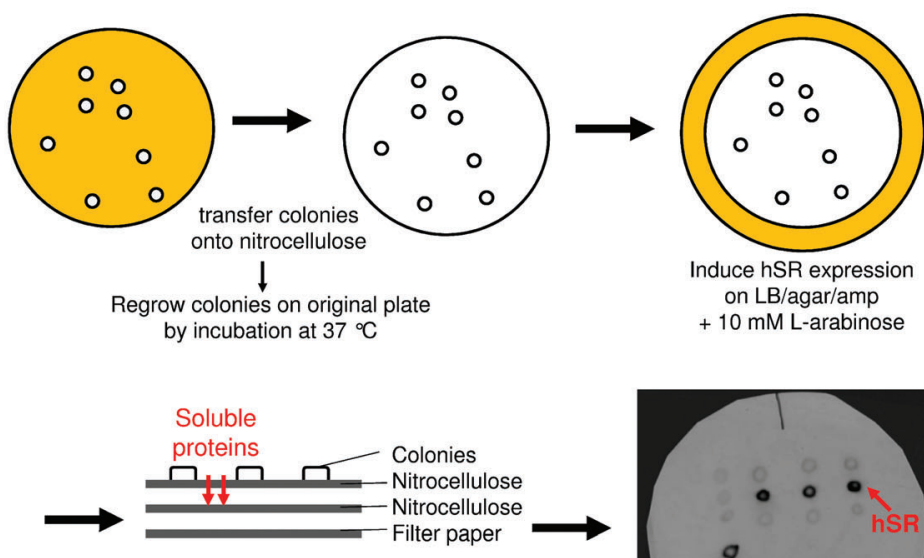

Set up a sandwich and lyse cells (lysozyme + freeze/thaw)

Soluble proteins diffuse to the lower nitrocellulose membrane

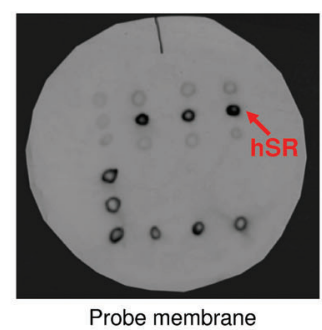

with hSR-specific antibodies

b

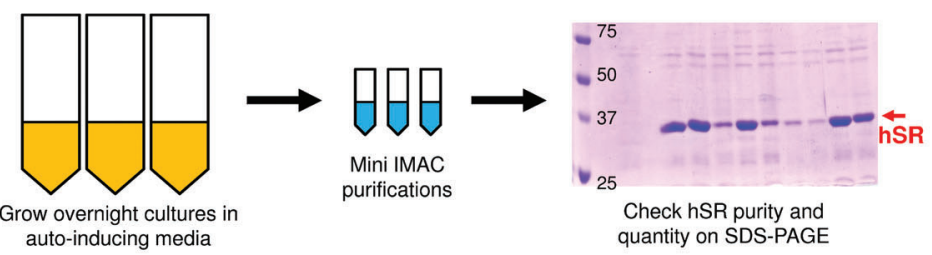

FIG. 1

Overview of the screening procedure. a Colony blot assay protocol, b Partial purification for activity measurements. Detailed procedures are given in Experimental 
Twenty-seven positive colonies were picked from the original plates and grown overnight in $10 \mathrm{ml}$ cultures of auto-inducing media. Small-scale purifications were carried out by immobilized metal affinity chromatography (IMAC) as shown in Fig. 1b. The IMAC-purified samples were then analyzed by SDS-PAGE to estimate hSR purity and quantity and subjected to activity analysis using our previously described HPLC-based endpoint assays $^{1,12}$. The activity reactions were carried out at $\mathrm{pH} 8.0$ in the presence of the SR activators ATP, $\mathrm{MgCl}_{2}$ and DTT, as described in Experimental. Both the L-serine racemization and elimination activities of the mutants were evaluated relative to wild-type hSR, and the results of the screening are shown in Table I and in Figs $2 \mathrm{a}$ and $2 \mathrm{~b}$. The mutants were divided into two

TABLE I

Summary of mutants identified by small-scale screening

Mutants with near-wild-type activity

Mutants with $<50 \%$ wild-type activity

$\mathrm{V} 12 \mathrm{~A}$

V138M

E129K/R277H/Q299H

S8Q

K14M/N172D/M 227L

L29M/K77R/V138E/I149T/E304V

T30S/M 150L/I236V/I308V

N35D/Y231C/K306E/K327S

I23V/S32G

V68G

\author{
E133G/L176P \\ G39S/D132V \\ V142A/I195V \\ S84G/P111L* \\ K201R
}

S8G/E98G/K114T/K225R/F260L/K327R

D11G/F43S/V68A

D112E/A120T/R253G
Mutants with $50-70 \%$ wild-type activity

\section{S302P}

L48P

I149T/Q272H

Q36L/R135H

E234G
Mutants with no measurable activity

K221E/T324A

V64I/G186S/G188S/E276K

C217S

K221E

Mutants are divided into the following 4 categories based on L-serine racemization and elimination activity: near-wild-type activity, 50-70\% wild-type activity, compromised activity ( $<50 \%$ of wild-type activity), and no measureable activity. Mutants selected for largescale expression and purification are underlined. * No measurable racemization activity, but elimination activity was observed. 
major categories - those that were active and those without measurable activity. The active mutants were further divided into three subcategories: mutants with near-wild-type activity, mutants that retained a substantial amount of activity (50-70\% of the wild-type values), and mutants with compromised activity ( $<50 \%$ of the wild-type values) (see Table I).

\begin{tabular}{|c|c|c|c|c|c|c|c|c|c|c|c|c|c|c|c|c|c|c|c|c|c|c|c|c|}
\hline 1 & 2 & 3 & 4 & 5 & 6 & 7 & 8 & 9 & 10 & 11 & 12 & 13 & 14 & 15 & 16 & 17 & 18 & 19 & 20 & 21 & 22 & 23 & 24 & 25 \\
\hline $\mathrm{M}$ & $\mathrm{C}$ & $\mathrm{A}$ & $\mathrm{Q}$ & $\mathrm{Y}$ & $\mathrm{C}$ & I & $\mathrm{S}$ & $\mathrm{F}$ & $\bar{A}$ & $\mathrm{D}$ & $\mathrm{V}$ & $\mathrm{E}$ & $\mathrm{K}$ & A & $\mathrm{H}$ & I & $\mathrm{N}$ & I & $\mathrm{R}$ & $\mathrm{D}$ & $\mathrm{S}$ & I & $\mathrm{H}$ & $\mathrm{L}$ \\
\hline & & & & & & & G & & & G & A & & $\mathrm{M}$ & & & & & & & & & V & & \\
\hline 26 & 27 & 28 & 29 & 30 & 31 & 32 & 33 & 34 & 35 & 36 & 37 & 38 & 39 & 40 & 41 & 42 & 43 & 44 & 45 & 46 & 47 & 48 & 49 & 50 \\
\hline $\mathrm{T}$ & $P$ & $\mathrm{~V}$ & $\mathrm{~L}$ & $\mathrm{~T}$ & $\mathrm{~S}$ & $\mathrm{~S}$ & I & $\mathrm{L}$ & $\mathrm{N}$ & $\mathrm{Q}$ & $\mathrm{L}$ & $\mathrm{T}$ & $\mathrm{G}$ & $\mathrm{R}$ & $\mathrm{N}$ & $\mathrm{L}$ & $F$ & $\mathrm{~F}$ & $\mathrm{~K}$ & $\mathrm{C}$ & $\mathrm{E}$ & $\mathrm{L}$ & $\mathrm{F}$ & $\mathrm{Q}$ \\
\hline & & & $\mathrm{M}$ & $\mathrm{S}$ & & G & & & D & L & & & $\mathrm{S}$ & & & & $\mathrm{S}$ & & & & & $\mathrm{P}$ & & \\
\hline 51 & 52 & 53 & 54 & 55 & 56 & 57 & 58 & 59 & 60 & 61 & 62 & 63 & 64 & 65 & 66 & 67 & 68 & 69 & 70 & 71 & 72 & 73 & 74 & 75 \\
\hline $\mathrm{K}$ & $\mathrm{T}$ & $\mathrm{G}$ & $\mathrm{S}$ & $\mathrm{F}$ & $\mathrm{K}$ & I & $\mathrm{R}$ & $\mathrm{G}$ & $\mathrm{A}$ & $\mathrm{L}$ & $\mathrm{N}$ & $\bar{A}$ & V & $\mathrm{R}$ & $\mathrm{S}$ & $\mathrm{L}$ & $\mathrm{V}$ & $\mathrm{P}$ & $\mathrm{D}$ & $\mathrm{A}$ & $\mathrm{L}$ & $\mathrm{E}$ & $\mathrm{R}$ & $\mathrm{K}$ \\
\hline & & & & & & & & & & & & & I & & & & A & & & & & & & \\
\hline & & & & & & & & & & & & & & & & & G & & & & & & & \\
\hline 76 & 77 & 78 & 79 & 80 & 81 & 82 & 83 & 84 & 85 & 86 & 87 & 88 & 89 & 90 & 91 & 92 & 93 & 94 & 95 & 96 & 97 & 98 & 99 & 100 \\
\hline $\mathrm{P}$ & $\mathrm{K}$ & $\mathrm{A}$ & $\mathrm{V}$ & $\mathrm{V}$ & $\mathrm{T}$ & $\mathrm{H}$ & $\mathrm{S}$ & $\mathrm{S}$ & $\mathrm{G}$ & $\mathrm{N}$ & $\mathrm{H}$ & $\mathrm{G}$ & $\mathrm{Q}$ & A & $\mathrm{L}$ & $\mathrm{T}$ & $Y$ & A & $\mathrm{A}$ & $\mathrm{K}$ & $\mathrm{L}$ & $\mathrm{E}$ & $\mathrm{G}$ & I \\
\hline & $\mathrm{R}$ & & & & & & & G & & & & & & & & & & & & & & G & & \\
\hline 101 & 102 & 103 & 104 & 105 & 106 & 107 & 108 & 109 & 110 & 111 & 112 & 113 & 114 & 115 & 116 & 117 & 118 & 119 & 120 & 121 & 122 & 123 & 124 & 125 \\
\hline $\mathrm{P}$ & $\mathrm{A}$ & $\mathrm{Y}$ & I & $\mathrm{V}$ & $\mathrm{V}$ & $\mathrm{P}$ & $\mathrm{Q}$ & $\mathrm{T}$ & $\mathrm{A}$ & $\mathrm{P}$ & $\mathrm{D}$ & $\mathrm{C}$ & $\mathrm{K}$ & $\mathrm{K}$ & $\mathrm{L}$ & $\mathrm{A}$ & I & Q & $\mathrm{A}$ & $\mathrm{Y}$ & $\mathrm{G}$ & $\mathrm{A}$ & $\mathrm{S}$ & I \\
\hline & & & & & & & & & & $\mathrm{L}$ & E & & $\mathrm{T}$ & & & & & & $\mathrm{T}$ & & & & & \\
\hline 126 & 127 & 128 & 129 & 130 & 131 & 132 & 133 & 134 & 135 & 136 & 137 & 138 & 139 & 140 & 141 & 142 & 143 & 144 & 145 & 146 & 147 & 148 & 149 & 150 \\
\hline $\mathrm{V}$ & $\mathrm{Y}$ & $\mathrm{C}$ & $E$ & $\mathrm{P}$ & $\mathrm{S}$ & D & $\mathrm{E}$ & $\mathrm{S}$ & $\mathrm{R}$ & $\mathrm{E}$ & $\mathrm{N}$ & $\mathrm{V}$ & $\mathrm{A}$ & $\mathrm{K}$ & $\mathrm{R}$ & $\mathrm{V}$ & $\mathrm{T}$ & $\mathrm{E}$ & $\mathrm{E}$ & $\mathrm{T}$ & $\mathrm{E}$ & $\mathrm{G}$ & I & $\mathrm{M}$ \\
\hline & & & $\mathrm{K}$ & & & V & G & & $\mathrm{H}$ & & & $\mathrm{M}$ & & & & A & & & & & & & $\mathrm{T}$ & $\mathrm{L}$ \\
\hline & & & & & & & & & & & & E & & & & & & & & & & & & \\
\hline 151 & 152 & 153 & 154 & 155 & 156 & 157 & 158 & 159 & 160 & 161 & 162 & 163 & 164 & 165 & 166 & 167 & 168 & 169 & 170 & 171 & 172 & 173 & 174 & 175 \\
\hline $\mathrm{V}$ & $\mathrm{H}$ & $\mathrm{P}$ & $\mathrm{N}$ & $\mathrm{Q}$ & $\mathrm{E}$ & $\mathrm{P}$ & A & $\mathrm{V}$ & I & $\mathrm{A}$ & $\mathrm{G}$ & $\mathrm{Q}$ & $\mathrm{G}$ & $\mathrm{T}$ & I & $\mathrm{A}$ & $\mathrm{L}$ & $\mathrm{E}$ & $\mathrm{V}$ & $\mathrm{L}$ & $\mathrm{N}$ & $\mathrm{Q}$ & $\mathrm{V}$ & $\mathrm{P}$ \\
\hline 176 & 177 & 178 & 179 & 180 & 181 & 182 & 183 & 184 & 185 & 186 & 187 & 188 & 189 & 190 & 191 & 192 & 193 & 194 & 195 & 196 & 197 & 198 & 199 & 200 \\
\hline L & $\mathrm{V}$ & $\mathrm{D}$ & $\mathrm{A}$ & $\mathrm{L}$ & $\mathrm{V}$ & $\mathrm{V}$ & $\mathrm{P}$ & $\mathrm{V}$ & $\mathrm{G}$ & $\mathrm{G}$ & $\mathrm{G}$ & $\mathrm{G}$ & $\mathrm{M}$ & $\mathrm{L}$ & A & $\mathrm{G}$ & I & A & I & $\mathrm{T}$ & $\mathrm{V}$ & $\mathrm{K}$ & A & $\mathrm{L}$ \\
\hline P & & & & & & & & & & $\mathrm{S}$ & & S & & & & & & & V & & & & & \\
\hline 201 & 202 & 203 & 204 & 205 & 206 & 207 & 208 & 209 & 210 & 211 & 212 & 213 & 214 & 215 & 216 & 217 & 218 & 219 & 220 & 221 & 222 & 223 & 224 & 225 \\
\hline $\mathrm{K}$ & $P$ & $\mathrm{~S}$ & $\mathrm{~V}$ & $\mathrm{~K}$ & $\mathrm{~V}$ & $\mathrm{Y}$ & $\mathrm{A}$ & $\mathrm{A}$ & $E$ & $\mathrm{P}$ & $\mathrm{S}$ & $\mathrm{N}$ & $\mathrm{A}$ & $\mathrm{D}$ & $\mathrm{D}$ & $\mathrm{C}$ & $\mathrm{Y}$ & $\mathrm{Q}$ & $\mathrm{S}$ & $\mathrm{K}$ & $\mathrm{L}$ & $\mathrm{K}$ & $\mathrm{G}$ & $\mathrm{K}$ \\
\hline $\mathrm{R}$ & & & & & & & & & & & & & & & & S & & & & E & & & & $\mathrm{R}$ \\
\hline 226 & 227 & 228 & 229 & 230 & 231 & 232 & 233 & 234 & 235 & 236 & 237 & 238 & 239 & 240 & 241 & 242 & 243 & 244 & 245 & 246 & 247 & 248 & 249 & 250 \\
\hline $\mathrm{L}$ & $\mathrm{M}$ & $\mathrm{P}$ & $\mathrm{N}$ & $\mathrm{L}$ & $Y$ & $\mathrm{P}$ & $P$ & $\mathrm{E}$ & $\mathrm{T}$ & I & $\mathrm{A}$ & $\mathrm{D}$ & $\mathrm{G}$ & $\mathrm{V}$ & $\mathrm{K}$ & $\mathrm{S}$ & $\mathrm{S}$ & I & $\mathrm{G}$ & $\mathrm{L}$ & $\mathrm{N}$ & $\mathrm{T}$ & $\mathrm{W}$ & $\mathrm{P}$ \\
\hline & $\mathrm{L}$ & & & & $\mathrm{C}$ & & & G & & V & & & & & & & & & & & & & & \\
\hline 251 & 252 & 253 & 254 & 255 & 256 & 257 & 258 & 259 & 260 & 261 & 262 & 263 & 264 & 265 & 266 & 267 & 268 & 269 & 270 & 271 & 272 & 273 & 274 & 275 \\
\hline I & I & $\mathrm{R}$ & $\mathrm{D}$ & $\mathrm{L}$ & $\mathrm{V}$ & $\mathrm{D}$ & $\mathrm{D}$ & I & $\mathrm{F}$ & $T$ & $\mathrm{~V}$ & $\mathrm{~T}$ & $\mathrm{E}$ & $\mathrm{D}$ & $\mathrm{E}$ & I & $\mathrm{K}$ & $\mathrm{C}$ & $\mathrm{A}$ & $\mathrm{T}$ & $\mathrm{Q}$ & $\mathrm{L}$ & $\mathrm{V}$ & $\mathrm{W}$ \\
\hline & & $\mathrm{G}$ & & & & & & & $\mathrm{L}$ & & & & & & & & & & & & $\mathrm{H}$ & & & \\
\hline 276 & 277 & 278 & 279 & 280 & 281 & 282 & 283 & 284 & 285 & 286 & 287 & 288 & 289 & 290 & 291 & 292 & 293 & 294 & 295 & 296 & 297 & 298 & 299 & 300 \\
\hline$E$ & $\mathrm{R}$ & $\mathrm{M}$ & $\mathrm{K}$ & $\mathrm{L}$ & $\mathrm{L}$ & I & $\mathrm{E}$ & $\mathrm{P}$ & $\mathrm{T}$ & $\mathrm{A}$ & $\mathrm{G}$ & $\mathrm{V}$ & G & $\mathrm{V}$ & $\mathrm{A}$ & $\mathrm{A}$ & $\mathrm{V}$ & $\mathrm{L}$ & S & Q & $\mathrm{H}$ & F & $\mathrm{Q}$ & $\mathrm{T}$ \\
\hline $\mathrm{K}$ & $\mathrm{H}$ & & & & & & & & & & & & & & & & & & & & & & $\mathrm{H}$ & \\
\hline 301 & 302 & 303 & 304 & 305 & 306 & 307 & 308 & 309 & 310 & 311 & 312 & 313 & 314 & 315 & 316 & 317 & 318 & 319 & 320 & 321 & 322 & 323 & 324 & 325 \\
\hline $\mathrm{V}$ & $\mathrm{S}$ & $\mathrm{P}$ & $\mathrm{E}$ & $\mathrm{V}$ & $\mathrm{K}$ & $\mathrm{N}$ & I & $\mathrm{C}$ & I & $\mathrm{V}$ & $\mathrm{L}$ & $\mathrm{S}$ & $\mathrm{G}$ & $\mathrm{G}$ & $\mathrm{N}$ & $\mathrm{V}$ & D & $\mathrm{L}$ & $\mathrm{T}$ & $\mathrm{S}$ & $\mathrm{S}$ & I & $\mathrm{T}$ & W \\
\hline & $\mathrm{P}$ & & V & & $\mathrm{E}$ & & V & & & & & & & & & & & & & & & & A & \\
\hline 326 & 327 & 328 & 329 & 330 & 331 & 332 & 333 & 334 & 335 & 336 & 337 & 338 & 339 & 340 & & & & & & & & & & \\
\hline $\mathrm{V}$ & $\mathrm{K}$ & $\mathrm{Q}$ & $\mathrm{A}$ & $\mathrm{E}$ & $\mathrm{R}$ & $P$ & A & $\mathrm{S}$ & $\mathrm{Y}$ & Q & $\mathrm{S}$ & $\mathrm{V}$ & $\mathrm{S}$ & $\mathrm{V}$ & & & & & & & & & & \\
\hline & $\begin{array}{l}\mathrm{R} \\
\mathrm{S}\end{array}$ & & & & & & & & & & & & & & & & & & & & & & & \\
\hline
\end{tabular}

\section{FIG. $2 a$}

Location of mutated sites in the hSR sequence. Primary amino acid sequence of human SR depicting all the mutation sites. Mutations occurring in SR variants that retained some activity in the initial screening experiments are highlighted yellow, while mutations occurring in inactive variants are highlighted green. Lysine 56 is in blue and residues coordinating $\mathrm{Mg}^{2+}$ are in red letters. 
To predict the positions of the mutations in the 3D structure, we analyzed a previously described homology model of hSR ${ }^{17}$. The majority of the mutations occurred at residues predicted to be at or near the surface of the enzyme, and a large percentage of the mutations occurred in putative loop regions (see Fig. 2 b). This observation is likely due to the fact that our screening procedure selects for soluble, well-expressing hSR variants. Amino acid substitutions in the enzyme core may result in more drastic structural changes, leading to insolubility or poor expression levels. To test this hypothesis, we selected 11 colonies with low or undetectable levels of soluble hSR expression for DNA sequencing. Of the 11 mutants, 2 contained frameshift mutations and 5 contained nonsense mutations. Three of the remaining 4 mutants had mutations at putative PLP binding residues. The final mutant contained 7 amino acid changes (G39V/L67F/V151A/K225R/ M227V/T285S/W325R), including several mutations of residues predicted to occur in the interior of the protein.
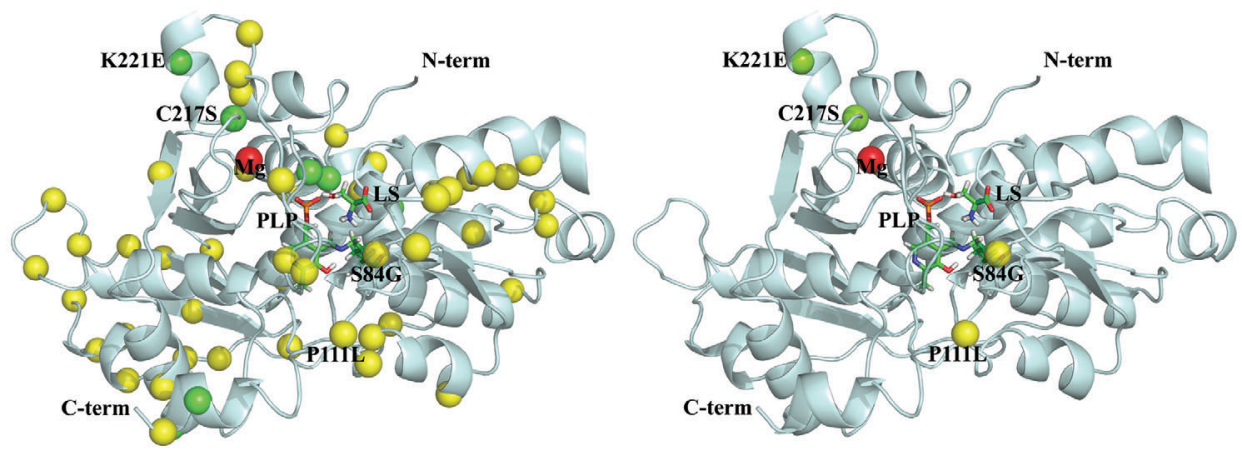

FIG. 2b

Location of mutated sites on the model. Homology model of hSR showing proposed locations of mutations. The picture on the left shows the positions of all observed mutations, and the picture on the right shows the positions of the studied mutations S84G, P111L, C217S, and K221E, which are labeled. Catalytically active mutations are shown as yellow spheres and inactive mutations as green spheres. PLP bound to Lys56 and the L-serine substrate are shown in sticks representation to enable visualization of the active site. The $\mathrm{Mg}^{2+}$ ion is shown as a red sphere. The figure was prepared with PyM ol ${ }^{35}$. The model depicted was built in Accelrys ${ }^{\mathrm{TM}}$ Discovery Studio 2.5 using the program MODELLER ${ }^{36}$ with the Schizosaccharomyces pombe SR structure $2 Z R 8{ }^{14}$ as a template. The modified PLP moiety [N-(5'-phosphopyridoxal)-D-alanine] present in the 2ZR8 structure was substituted in the homology model with unmodified PLP COvalently bound to the $\mathrm{N} \varepsilon$ atom of Lys56 by manual docking. The homology model of hSR was completed by adding the L-serine substrate and $\mathrm{Mg}^{2+}$ ion based on superposition with the 2ZR8 structure. Further computational refinement of the model was performed with AMBER 10 software $^{37}$ (unpublished data) 
Based on our results from this limited screening, hSR seems to be remarkably tolerant of surface mutations. Several variants containing multiple mutations (up to 5 in the case of L29M/K77R/V138E/I49T/E304V) retained near-wild-type racemization and elimination activity. Even a SR variant bearing 6 mutations (S8G/E98G/K114T/K225R/F260L/K327R) was soluble and showed measurable serine racemization and elimination activity. It has been well-documented that mutations in surface patches can influence an enzyme ability to crystallize $\mathrm{e}^{25,26}$, and the random mutagenesis strategy outlined in this work could be extended in the future to identify hSR variants with an enhanced ability to form crystal contacts.

Few soluble mutants that effectively lacked both racemization and elimination activity were identified. The V64I/G186S/G188S/E276K mutant contains two mutations in the tetraglycine loop (Gly185-Gly188 in hSR), a common motif in PLP-dependent enzymes that stabilizes the phosphate moiety of the PLP cofactor. Destabilization of the PLP cofactor is a likely explanation for the activity loss. The K221E/T324A and K221E mutants were both inactive, pointing to the significance of Iysine 221 for SR activity. The C217S hSR mutant was also inactive; C217S mouse SR has been previously reported to lack racemase activity, though the authors did not speculate about the reason for the activity $\operatorname{loss}^{27}$. We found the substantial activity loss brought about by the K221E and C217S single mutations intriguing, particularly since both residues are in the vicinity of the $\mathrm{Mg}^{2+}$ coordinating residues (Fig. 3).

The S84G/P111L mutant was somewhat of an outlier in the screening assay, since its racemization activity was more dramatically affected than its elimination activity. Furthermore, the S84G mutation is predicted to occur not at the surface of the molecule but rather within the active site of SR (see Fig. 2b). We set out to study these three interesting mutants - C217S, $\mathrm{K} 221 \mathrm{E}$, and the double mutant S84G/P111L - in more detail.

Large Scale Expression and Purification of W ild-Type,

C217S, K211E, and S84G/P111L hSR

In order to produce a sufficient amount of protein for more detailed functional studies, we scaled up the expression of wild-type, C217S, K221E, and S84G/P111L His-tagged hSR (1.5-3 I of culture). Highly purified proteins were obtained by FPLC-assisted IMAC purification as described in Experimental. A yield of $0.8 \mathrm{mg}$ purified wild-type hSR was obtained per liter of culture. Yields of the C217S, K221E, and S84G/P111L mutants were 1.52, 0.8 , and $1.34 \mathrm{mg} / \mathrm{l}$, respectively. The successful upscaled purification of 


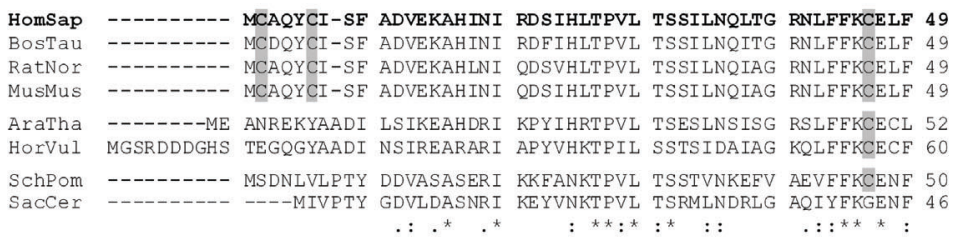

HOMSAP QKTGSFKIRG ALNAVRSLVP DALERKPKAV VTHSSGNHGQ ALTYAAKLEG IPAYIVVPQT 109 BosTau QKTGSEKIRG ALNAIRGLIS AHPEEKPRAV VAHSSGNHGQ ALSFAARLEG IPAYVIVPET 109 RatNor QKTGSEKIRG ALNAIRGLIP DTLEGKPKAV VTHSSGNHGQ ALTYAAKLEG IPAYIVVPQT 109 MusMuS QKTGSEKIRG ALNAIRGLIP DTPEEKPKAV VTHSSGNHGQ ALTYAAKLEG IPAYIVVPQT 109 AraTha QKGGAFKERG ACNAVLSLD- -AEQAA-KGV VTHSSGNHAA ALSLAAKIQG IPAYIVVPKG 109 HorVul OKAGAFKIRG ASNSIEALD- -DSQAA-KGV VTHSSGNHAA AVALAAKLRG IPAYIVIPKN 117 SchPom QKMGAEKERG ALNALSQLN- -EAQRK-AGV LTFSSGNHAQ AIALSAKILG IPAKIIMPLD 107 SacCer QRVGAFKERG AMNAVSKLS- -DEKRS-KGV IAFSSGNHAQ AIALSAKLLN VPATIVMPED 103

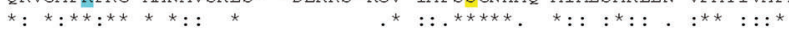

HomSap APDCKKLAIQ AYGASIVYCE PSDESRENVA KRVTEETEGI MVHPNQE PAV IAGQGTIALE 169 BOSTau APNCKKLAIQ AYGASIVYSE QSEESRENIT KRIAEETEGI MVHPNQEPAV IAGQGTIAME 169 RatNor APNCKKLAIQ AYGASIVYSE PSDESRENVA QRIIQETEGI LVHPNQEPAV IAGQGTIALE 169 MusMus APNCKKLAIQ AYGASIVYCD PSDESREKVT QRIMQETEGI LVHPNQEPAV IAGQGTIALE 169 AraTha APKCKVDNVI RYGGKVIWSE ATMSSREEIA SKVLQETGSV LIHPYNDGRI ISGQGTIALE 169 HorVuI APACKVENVR RYGGQVIWSD VTMESRESIA KKVQEETGAI LIHPFNDKYT ISGQGTVCLE 177 SchPOM APEAKVAATK GYGGQVIMYD RYKDDREKMA KEISEREGLT IIPPYDHPHV LAGQGTAAKE 167 SacCer APALKIAATA GYGAHIIRYN RYTEDREQIG RQLAAEHGFA LIPPYDHPDV IAGQGTSAKE 163

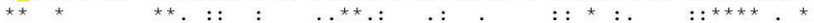

HOMSAP VLNQVPLVDA LVVPVGGGGM LAGIAITVKA LKPSVKVYAA EPSNADDCYQ SKLKGKLMPN 229 BosTau VLNQVPLVDA LVVPVGGGGM LAGIAVTVKA LRPSVKVYAA EPLNADDCYQ SKLKGELTPN 229 RatNor VLNQVPLVDA LVVPVGGGGM VAGIAITIKT LKPSVKVYAA EPSNADDCYQ SKLKGELTPN 229 MusMus VLNQVPLVDA LVVPVGGGGM VAGIAITIKA LKPSVKVYAA EPSNADDCYQ SKLKGELTPN 229 AraTha LLEQIQEIDA IVVPISGGGL ISGVALAAKS IKPSIRIIAA EPKGADDAAQ SKVAGKII-T 228 HorVuI LLEQVPEIDT IIVPISGGGL ISGVTLAAKA INPSIRILAA EPKGADDSAQ SKAAGRII-K 236 SchPom LEEEVGPLDA LEVCLGGGGL LSGSALAARH EAPNCEVYGV EPEAGNDGQQ SFRKGSIV-H 226 SacCer LLEEVGQLDA LEVPLGGGGL LSGSALAARS LSPGCKIFGV EPEAGNDGQQ SERSGSIV-H 222

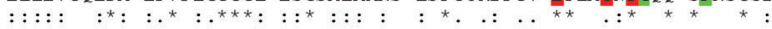

HOMSAP LYPPETIADG VKS-SIGLNT WPIIRDLVDD IFTVTEDEIK CATQLVWERM KLIIEPTAGV 288 BOSTau PYPPETIADG IKS-SIGLNT WPIIRDLVDD VETVTEDEIK YATQLVWERM KLLIEPTAGV 288 RatNor LHPPETIADG VKS-SIGLNT WPIIRDLVDD VETVTEDEIK YATQLVWERM KLLIEPTAGV 288 MUSMUS LHPPETIADG VKS-SIGLNT WPIIRDLVDD VETVTEDEIK YATQLVWGRM KLLIEPTAGV 288

AraTha LPVTNTIADG LRA-SLGDLT WPVVRDLVDD VVTLEECEII EAMKMCYEIL KVSVEPSGAI 287 HorVul LPATSTIADG LRA-FLGDLT WPVVRDLVDD VIVVDDNAIV DAMKMCYETL KVAVEPSGAI 295 SChPOM IDTPKTIADG AQTQHLGNYT FSIIKEKVDD ILTVSDEELI DCLKFYAARM KIVVEPTGCL 286 SaCCE INTPKTIADG AQTQHLGEYT FAIIRENVDD ILTVSDQELV KCMHFLAERM KVVVEPTACL 282

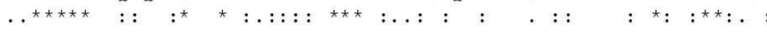

HOMSAP GVAAVLSQHF QTVSPEV--K NICIVLSGGN VDLTSSITWV KQ-AERPASY QSVSV 340 BosTau GVAVVLSQHF RTVPAEV--K NICIVLSGGN VDLTS-LTWV KKQDEKAAP- ---- 334 RatNor GLAAVLSQHF QTVSPEV--K NICIVLSGGN VDLTS-LSWV K-QAERPAP- ----- 333 MusMus ALAAVLSQHF QTVSPEV--K NVCIVLSGGN VDLTS-LNWV G-QAERPAPY QTVSV 339 AraTha GLAAVLSNSF RNNPSCRDCK NIGIVLSGGN VDLGS--LWD SFKSSK---- ----- 331 HorVul GLAAALSDEF KQSSAWHESS KIGIIVSGGN VDLRV--LWD SLYK------ ----- 337

SChPOM SFAAARAMKE -----KLKNK RIGIIISGGN VDIERYAHFL SQ-------- ---- 323 SacCer GEAGALLKKE -----ELVGK KVGIILSGGN VDMKRYATLI SGKEDGPTI- -- - - 326

\section{FIG. 3}

Multiple sequence alignment of 8 eukaryotic serine racemases from Homo sapiens (HomSap), Bos Taurus (BosTau), Rattus norvegicus (RatNor), Mus musculus (MusMus), Arabidopsis thaliana (AraTha), Hordeum vulgare (HorVul), Schizosaccharomyces Pombe (SchPom), and Saccharomyces cerevisiae (SacCer). The sites of mutations S84G, P111L, C217S, and K221E are in yellow or in green (see legend to Fig. 2a); PLP binding Lys56 is in blue; residues coordinating $\mathrm{Mg}^{2+}$ are in red; hSR cysteines as well as conserved cysteines of other SR orthologues are in grey 
these three mutants suggests that the results of our small-scale screening procedure are easily extendable to larger-scale applications.

All of the mutants contained an active-site-bound PLP based on spectrophotometric analysis. The oligomerization state of wild-type SR and the mutants was accessed by gel filtration on Superdex200HR as described in Experimental. The wild-type, K221E, and C217S mutants eluted as a single major peak corresponding to roughly $60 \mathrm{kDa}$, which is in accord with the results of Dunlop and Neidle ${ }^{3}$. While this is slightly lower than the predicted molecular weight of a SR dimer ( $74 \mathrm{kDa})$, it is significantly larger than the molecular weight of the monomer ( $37 \mathrm{kDa})$, and SR has been reported to form homodimers by several groups ${ }^{1,3,5}$. Thus, we presume that wild-type, C217S, and K221E hSR are present in solution primarily in dimeric form. The S84G/P111L mutant also predominantly formed dimers, but significant amounts of higher molecular weight species (115 and $230 \mathrm{kDa}$ ) that might correspond to tetrameric and octameric species were also present.

\section{Thermal Stability of C217S, K221E, and S84G/P111L hSR}

The thermal stabilities of wild-type hSR and the C217S, K221E, and S84G/P111L mutants were assessed using ThermoFluor, a biophysical technique for determination of relative protein stabilities based on fluorophore partition into the denatured protein upon temperature increase ${ }^{28-30}$. In the absence of SR activators, wild-type, C217S, and K221E hSR exhibited melting temperatures of $63.2 \pm 0.53,63.1 \pm 0.17$, and $62.7 \pm 0.32{ }^{\circ} \mathrm{C}$, respectively. The S84G/P111L double mutant was slightly less stable, with $\mathrm{T}_{\mathrm{m}}=$ $61.6 \pm 0.40^{\circ} \mathrm{C}$.

As illustrated in Fig. 4, addition of the known SR activators $\mathrm{MgCl}_{2}$ and ATP (1 $\mathrm{mm}$ each) did not dramatically affect the melting temperature of wild-type or S84G/P111L hSR. On the other hand, the C217S and K221E mutants were stabilized by approximately $0.7{ }^{\circ} \mathrm{C}$. Addition of $\mathrm{MgCl}_{2}$ alone to these mutants had a similar effect, while addition of ATP alone did not cause significant stabilization (data not shown), suggesting that these mutants might be deficient in bound $\mathrm{Mg}^{2+}$. Assessment of the thermal stability of the SR variants in the presence of $5 \mathrm{mM}$ EDTA provided additional support for this hypothesis. Wild-type hSR and the S84G/P111L mutant were greatly destabilized by addition of EDTA with $\Delta \mathrm{T}_{\mathrm{m}}=-2.37$ and $-3.54{ }^{\circ} \mathrm{C}$, re spectively. C217S and K221E hSR were only moderately affected with $\Delta \mathrm{T}_{\mathrm{m}}=$ -0.92 and $-0.41{ }^{\circ} \mathrm{C}$, respectively (see Fig. 4).

The C217S and K221E mutants also showed altered sensitivity to reducing agents compared to wild-type and S84G/P111L hSR. Addition of $5 \mathrm{~mm}$ 
DTT had a strong stabilizing effect on wild-type and the double mutant $\left(\Delta T_{m}=3.4\right.$ and $3.2{ }^{\circ} \mathrm{C}$, respectively, compared to buffer alone). A comparable stabilizing effect could be achieved by addition of $2 \mathrm{~mm}$ tris(2-carboxylethyl)phosphine or $20 \mathrm{~mm} \beta$-mercaptoethanol (data not shown). The requirement of free thiol groups for SR activity has been noted ${ }^{18}$, but the mechanism of SR stabilization and activation in reducing conditions is not yet understood. Human SR is a cysteinerich protein containing 8 cysteine residues per monomer, yet there is no evidence indicating that disulfide bridges are present in the enzyme's native conformation. In fact, 5 of the cysteines are predicted to be surface-exposed. Sulfur in cysteine can adopt several redox states, and Cys is therefore involved in a number of functions and it is a common site for posttranslational modifications ${ }^{31}$. In the case of mouse SR, Mustafa et al. ${ }^{27}$ have shown that modification at C113 can prevent ATP binding and activation. Addition of reducing agents to SR may prevent unwanted modifications and lead to protein stabilization by keeping the exposed cysteines in a reduced state. Another role for reducing agents could be prevention of irreversible oxidation of the enzyme. A recent review indicates that some PLP-containing enzymes can undergo paracatalytic side reactions with molecular oxygen or ${ }^{*} \mathrm{NO}$ radical, leading to

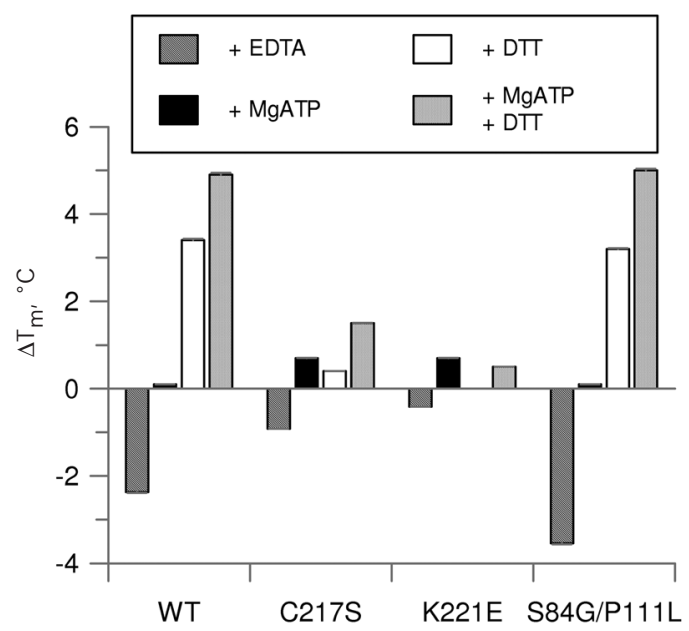

FIG. 4

Melting temperature changes $\left(\Delta \mathrm{T}_{\mathrm{m}},{ }^{\circ} \mathrm{C}\right)$ in the presence of various SR cofactors and additives. All samples contained 100 mM HEPES (pH 8.0) and $10 \mu \mathrm{M}$ PLP. Samples labeled "+EDTA" con-

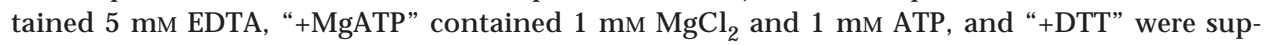
plemented with $5 \mathrm{~mm}$ DTT 
deactivation of the enzyme ${ }^{32}$. This mechanism of inactivation has been described for some decarboxylases, and it can be prevented by addition of thiols.

In contrast to the wild-type, the stabilities of the C217S and K221E mutants were not significantly affected by addition of DTT, which might suggest that these mutants are structurally different, perhaps due to their impaired $\mathrm{Mg}^{2+}$ binding abilities. Cook et al. ${ }^{5}$ have shown that the fluorescence spectra of EDTA-treated $\mathrm{mSR}$ and $\mathrm{Ca}^{2+}{ }^{+}$-saturated mouse SR are markedly different, suggesting that binding of the divalent cation causes a dramatic structural change. In almost all cases, the exception being the K221E mutant, the hSR variants exhibited the highest $T_{m}$ values in the presence of all three activators, with wild-type being the most stable, followed by S84G/P111L, then C217S and K221E.

Kinetic Characterization of C217S, K221E, and S84G/P111L hSR

Since the thermal stability analyses indicated that the C217S and K221E mutants were differently affected by the presence of reducing agents than the S84G/P111L mutant and wild-type SR, we measured the specific activities of the purified SR variants in the presence and absence of $5 \mathrm{~mm}$ DTT. We have previously observed that wild-type SR purified and stored with a high concentration of DTT is not significantly affected by addition of DTT to the activity reaction, while SR purified and stored with low levels of DTT is activated by addition of DTT (unpublished results). To probe the effects of high concentrations of DTT on activity of the mutants, we stored the mutants and the wild-type in the presence of a low concentration of DTT $(0.1 \mathrm{~mm})$. All activity reactions were conducted in the presence of $1 \mathrm{~mm}$ $\mathrm{MgCl}_{2}, 1 \mathrm{mM}$ ATP, and $10 \mu \mathrm{M}$ PLP. Working with high concentrations of the purified proteins, we were able to detect a low level of serine racemization for all three mutants (Figs $5 a$ and $5 b$ ). Wild-type SR was strongly activated in the presence of $5 \mathrm{~mm}$ DTT, with specific activity values 6- and 4-fold higher in the presence of DTT than in its absence for the L-serine to D-serine (Fig. 5a) and D-serine to L-serine (Fig. 5b) conversions, respectively. In contrast, none of the mutants were significantly activated by DTT.

The conversion of D-serine to pyruvate (Fig. $5 d$ ) is the least efficient of the four serine reactions catalyzed by SR, but it seems to be the most affected by DTT. The wild-type specific activity was 9-fold higher in the presence of DTT than in its absence, and all of the mutants were slightly activated (2- to 3-fold) by addition of DTT. As with the racemization reac- 
tions, all of the mutants exhibited severely compromised activity compared to the wild-type.

A slightly different pattern was observed for the L-serine to pyruvate conversion (Fig. 5c). Again, all of the mutants are less active than wild-type hSR. The wild-type is activated 4-fold by addition of $5 \mathrm{~mm}$ DTT, while the C217S and K221E mutants exhibited compromised activity that could not be recovered by addition of DTT. However, in this case, the S84G/P111L mutant shows a 3-fold increase in specific activity upon addition of DTT. Recently, Goto et al. ${ }^{14}$ produced an S82A mutant of S. pombe SR (S82 corresponds to S84 of hSR), which reportedly lost racemization and D-serine dehydratase activity but retained a substantial amount of L-serine dehydratase activity (about $50 \%$ of the wild-type level). In contrast to their results, we observe that, even when activated by addition of DTT, the S84G/P111L hSR mutant still exhibits compromised L-serine dehydratase activity (about 10\% of the wild-type value). Furthermore, we observe that S84G/P111L hSR

a

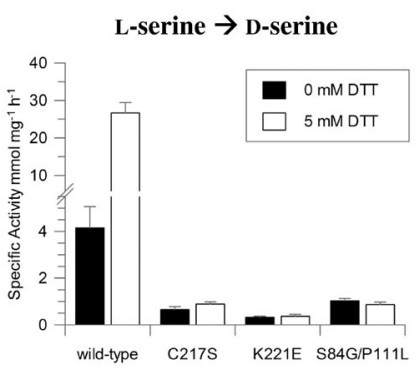

C

L-serine $\rightarrow$ pyruvate

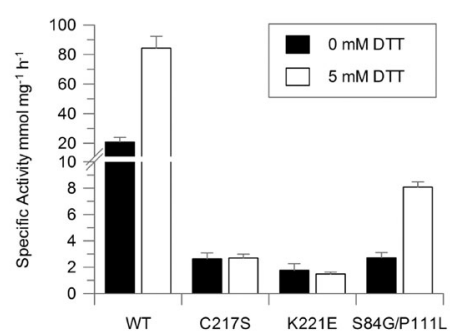

b

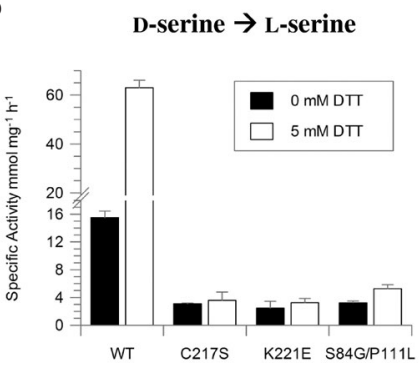

d D-serine $\rightarrow$ pyruvate

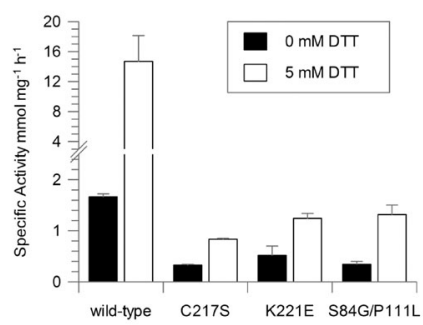

FIG. 5

Specific activity measurements. All reactions were conducted in the presence of $100 \mathrm{mM} H E P E S$ (pH 8.0), containing $1 \mathrm{~mm}$ PLP, $1 \mathrm{~mm} \mathrm{MgCl}_{2}$, and $1 \mathrm{~mm}$ ATP. Reaction mixtures were accessed by HPLC-based endpoint assay as described in Experimental. Specific activities are given in $\mathrm{mmol}$ of product evolved per $\mathrm{mg}$ of SR per hour. a L-serine to D-serine, b D-serine to L-serine, C L-serine to pyruvate, and d D-serine to pyruvate conversions 
has detectable, albeit very modest, racemization and D-serine elimination activity. However, a direct comparison between S82A S. pombe SR and S84G/P111L hSR is not a fair one, since the Ser to Gly mutation is more extreme than the Ser to Ala mutation. Furthermore, we speculate that the additional mutation at P111 might cause further changes to the enzyme.

\section{Multiple Alignment Analysis}

We created a multiple sequence alignment of eight eukaryotic racemases (from Homo sapiens, Bos taurus, Rattus norvegicus, Mus musculus, Arabidopsis thaliana, Hordeum vulgare, Saccharomyces cerevisiae, and Schizosaccharomyces pombe) shown in Fig. 3. The alignment resembles that prepared by Baumgart et al. ${ }^{18}$, who compared human, plant, and yeast SRs with aspartate racemase from the bivalve mollusk Scapharca broughtonii and SR from the bacteria Pyrobaculum islandicum.

All eight homologues included in our alignment catalyze both serine racemization and elimination and are activated by $\mathrm{Mg}^{2+}$ ions. Most of these racemases are also activated by ATP, with the exception of SR orthologues from Hordeum vulgare (barley) ${ }^{33}$ and Arabidopsis thaliana ${ }^{34}$. Mammalian SRs exhibit a very high degree of sequence identity (84-91\%), and we recently published a comparative study of the human and mouse orthologues ${ }^{17}$. Plant and yeast SRs boast a high level of sequence similarity to their mammalian orthologues (35-48\% identity). Plant SRs have a 2-10 amino acid extension at the $\mathrm{N}$-terminus, while both yeast and plant SRs are C-terminally truncated by 5-12 residues as compared to hSR. Predicted $\mathrm{Mg}^{2+}$-binding residues are conserved throughout the family (residues depicted in red in Figs 2 and 3).

The only available experimentally determined structures of a eukaryotic serine racemase are four crystal structures of SR from fission yeast, $\mathrm{S}$. pombe (PDB accession codes 2ZR8, 2ZPU, 1WTC, and 1V71)14,16. Like hSR, S. pombe SR acts as both a racemase and an eliminase and is activated by divalent cations and ATP. Several homology models of mammalian SRs have been created based on these structures ${ }^{15,17}$, and these models allow us to speculate about some features of the hSR structure and the potential structural effects of the mutations.

Cysteine 217 is conserved in mammalian SRs, while in yeast SRs the corresponding position is occupied by glycine. In SR from Arabidopsis thaliana the corresponding residue is an alanine, and in SR from Hordeum vulgare the position is occupied by a serine residue (see alignment in Fig. 3). In terms of sequence, Cys217 closely follows the magnesium coordinating residues 
Glu210, Ala214, and Asp216. Conceivably, a mutation at residue 217 may disrupt the $\mathrm{Mg}^{2+}$ coordination site by altering intramolecular interactions in the vicinity.

Lys221 is also sequentially close to $\mathrm{Mg}^{2+}$ coordinating residues. Lys221 is conserved among mammalian SRs, while plant SRs bear arginine and yeast SRs glutamine in the corresponding position (see Fig. 3). The K221E mutation leads to reversal of the residue charge and might cause loss of a strong and possibly important salt-bridge interaction, leading to disruption of the coordination site topology.

Unlike the C217S and K221E mutants, the S84G/P111L mutant harbors mutations at two highly conserved residues. Ser84 is predicted to be located within the active site as part of a so-called "asparagine loop" (Ser83-His87), which has been shown to participate in substrate recognition and catalysis in S. pombe SR ${ }^{14}$. Thus, the S84G mutation in hSR likely directly affects the active site. The $P 111 \mathrm{~L}$ mutation is predicted to occur within a short distance of the active site (Fig. 2b). P111 might be important for determining the correct orientation of the $\alpha$-helix spanning residues Asp112-Tyr121 due to proline's kink-forming propensity. It is possible that the proline to leucine mutation allows more flexibility in the orientation of the $\alpha$-helix, which may in turn affect the active site topology and contribute to the impaired activity of the double mutant. In the absence of an experimentally determined mammalian SR structure, it is difficult to understand the observed change in the multimerization state of this mutant compared to the wild-type. The SR dimerization domain has been predicted based on the S. pombe SR structure ${ }^{15}$, but a potential tetramerization domain has not been identified. It is attractive to speculate that the residues close to the active site might control multimerization of the protein or that the multimerization state might affect the enzymatic activity. The behavior of the double mutant compared to the wild-type on the gel filtration column is the first evidence that the multimerization equilibria of SR can be shifted. More detailed structural studies will be needed to clarify the relationship, if any, between SR activity and multimeric state.

\section{CONCLUSIONS}

Random mutagenesis is a valuable toolbox for the biochemist. In this work, we describe a method for the generation and screening of random human serine racemase mutants. We identified and screened $27 \mathrm{hSR}$ variants on a small scale, and we note, based on homology models, that the majority of the mutated sites are predicted to occur at or near the surface of the mole- 
cule. This suggests that the method outlined here could be applied to the identification of active hSR mutants with altered surface properties, which in turn can be applied to the search for an hSR variant that forms welldiffracting crystals. In this work, we apply the method to the structureactivity analysis of SR by selecting 3 interesting mutants for large-scale expression and purification and a more detailed functional analysis. We conclude that neighboring residues $\mathrm{C} 217$ and K221, which are moderately conserved among eukaryotic SRs, are important for the racemization and elimination activities of hSR as well as the sensitivity of the enzyme to $\mathrm{Mg}^{2+}$ and reducing agents. The S84G/P111L mutant exhibits a thermal stability profile close to that of the wild-type, but both its racemization and elimination activities are severely impaired, suggesting that one or both of these residues might be directly involved in catalysis. Furthermore, the S84G/P111L mutant displays an altered multimerization state, suggesting the interesting possibility that multimerization plays a role in activity regulation.

Note added in the proof

In the time period between acceptance and publication of this manuscript, the human serine racemase $X$-ray structure was reported (Smith M. A., Mack V., Ebneth A., Moraes I., Felicetti B., Wood M., Schonfeld D., Mather O., Cesura A., Barker J.: J. Biol. Chem., Epub ahead of print, doi:10.1074/jbc.M 109.050062).

We would like to thank I. Nováková for her help with the screening and K. Grantz-Šašková, J. Brynda, and M. Lepšík for valuable discussions. W e are grateful for the financial support provided by the Ministry of Education, Youth and Sports of the Czech Republic (Grant 1M 0508).

\section{REFERENCES}

1. Strisovsky K., Jiraskova J., Barinka C., Majer P., Rojas C., Slusher B. S., Konvalinka J.: FEBS Lett. 2003, 535, 44.

2. De Miranda J., Panizzutti R., Foltyn V. N., Wolosker H.: Proc. Natl. Acad. Sci. U.S.A. 2002, 99, 14542.

3. Neidle A., Dunlop D. S.: Neurochem. Res. 2002, 27, 1719.

4. Foltyn V. N., Bendikov I., De Miranda J., Panizzutti R., Dumin E., Shleper M., Li P., Toney M. D., Kartvelishvily E., Wolosker H.: J. Biol. Chem. 2005, 280, 1754.

5. Cook S. P., Galve-Roperh I., Martinez del Pozo A., Rodriguez-Crespo I.: J. Biol. Chem. 2002, 277, 27782.

6. Wolosker H., Sheth K. N., Takahashi M., Mothet J. P., Brady R. O., Jr., Ferris C. D., Snyder S. H.: Proc. Natl. Acad. Sci. U.S.A. 1999, 96, 721. 
7. Wu S. Z., Bodles A. M., Porter M. M., Griffin W. S., Basile A. S., Barger S. W.: J. Neuroinflammation 2004, 1, 2.

8. Sasabe J., Chiba T., Yamada M., Okamoto K., Nishimoto I., Matsuoka M., Aiso S.: EMBO J. 2007, 26, 4149.

9. Parsons C. G., Stoffler A., Danysz W.: Neuropharmacology 2007, 53, 699.

10. Lipton S. A.: Nat. Rev. Drug Discov. 2006, 5, 160.

11. Inoue R., Hashimoto K., Harai T., Mori H.: J. Neurosci. 2008, 28, 14486.

12. Strisovsky K., Jiraskova J., Mikulova A., Rulisek L., Konvalinka J.: Biochemistry 2005, 44, 13091.

13. Hoffman H. E., Jiraskova J., Cigler P., Sanda M., Schraml J., Konvalinka J.: J. Med. Chem. 2009, 52, 6032 .

14. Goto M., Yamauchi T., Kamiya N., Miyahara I., Yoshimura T., Mihara H., Kurihara T., Hirotsu K., Esaki N.: J. Biol. Chem. 2009, 284, 25944.

15. Baumgart F., Mancheno J. M., Rodriguez-Crespo I.: FEBS J. 2007, 274, 4561.

16. Yamauchi T., Goto M., Wu H. Y., Uo T., Yoshimura T., Mihara H., Kurihara T., Miyahara I., Hirotsu K., Esaki N.: J. Biochem. 2009, 145, 421.

17. Hoffman H. E., Jiraskova J., Ingr M., Zvelebil M., Konvalinka J.: Protein Expr. Purif. 2009, 63,62 .

18. Baumgart F., Rodriguez-Crespo I.: FEBS J. 2008, 275, 3538.

19. Tarun A. S., Lee J. S., Theologis A.: Proc. Natl. Acad. Sci. U.S.A. 1998, 95, 9796.

20. Spee J. H., de Vos W. M., Kuipers O. P.: Nucleic Acids Res. 1993, 21, 777.

21. Cornvik T., Dahlroth S. L., Magnusdottir A., Flodin S., Engvall B., Lieu V., Ekberg M., Nordlund P.: Proteins 2006, 65, 266.

22. Studier F. W.: Protein Expr. Purif. 2005, 41, 207.

23. Notredame C., Higgins D. G., Heringa J.: J. Mol. Biol. 2000, 302, 205.

24. Rasila T. S., Pajunen M. I., Savilahti H.: Anal. Biochem. 2009, 388, 71.

25. Derewenda Z. S.: Methods 2004, 34, 354.

26. Price S. R., Nagai K.: Curr. Opin. Biotechnol. 1995, 6, 425.

27. Mustafa A. K., Kumar M., Selvakumar B., Ho G. P., Ehmsen J. T., Barrow R. K., Amzel L. M., Snyder S. H.: Proc. Natl. Acad. Sci. U.S.A. 2007, 104, 2950.

28. Nettleship J. E., Brown J., Groves M. R., Geerlof A.: Methods Mol. Biol. 2008, 426, 299.

29. Pantoliano M. W., Petrella E. C., Kwasnoski J. D., Lobanov V. S., Myslik J., Graf E., Carver T., Asel E., Springer B. A., Lane P., Salemme F. R.: J. Biomol. Screen. 2001, 6, 429.

30. Lo M. C., Aulabaugh A., Jin G., Cowling R., Bard J., Malamas M., Ellestad G.: Anal. Biochem. 2004, 332, 153.

31. Jacob C., Giles G. I., Giles N. M., Sies H.: Angew. Chem. Int. Ed. 2003, 42, 4742.

32. Bunik V. I., Schloss J. V., Pinto J. T., Gibson G. E., Cooper A. J.: Neurochem. Res. 2007, $32,871$.

33. Fujitani Y., Horiuchi T., Ito K., Sugimoto M.: Phytochemistry 2007, 68, 1530.

34. Fujitani Y., Nakajima N., Ishihara K., Oikawa T., Ito K., Sugimoto M.: Phytochemistry 2006, 67, 668 .

35. DeLano W. L.: The PyMol Molecular Graphics System. DeLano Scientific, San Carlos (CA) 2002.

36. Eswar N., Marti-Renom M. A., Webb B., Madhusudhan M. S., Eramian D., Shen M., Pieper U., Sali A.: Current Protocols in Bioinformatics, Supplement 15. John Wiley \& Sons, New York 2006. 
37. Case D. A., Darden T. A., Cheatham III T. E., Simmerling C. L., Wang J., Duke R. E., Luo R., Crowley M., Walker R. C., Zhang W., Merz K. M., Wang B., Hayik S., Roitberg A., Seabra G., Kolossváry I., Wong K. F., Paesani F., Vanicek J., Wu X., Brozell S. R., Steinbrecher T., Gohlke H., Yang L., Tan C., Mongan J., Hornak V., Cui G., Mathews D. H., Seetin M. G., Sagui C., Babin V., Kollman P. A.: AMBER 10. University of California, San Francisco 2008. 